\title{
UNTERSUCHUNGEN ÜBER DIE NORDOST - ANATOLISCHEN FLECHTEN LOBARIA SCROBICULATA UND L. PULMONARIA
}

\section{Studies on the Northeast Anatolian lichens Lobaria scrobiculata and L. pulmonaria}

\section{U. Zeybek ${ }^{1}$ A. Yuldız ${ }^{2}$}

1-Ege Universität, Fakultät für Pharmazie, Abteilung für Pharmazeutische Botanik, 35100 BornovaIzmir, Türkei

2-Ankara Universität, Naturwissenschaftliche Fakultät, Abteilung Biologie, 06100 Tandogan Ankara, Türkei

(Keywords: Lichens, Lobaria, lichen substances, HPLC)

Abstract:

The secondary metabolites in Lobaria pulmonaria and L. scrobiculata collected in northeast Anatolia are studied using HPLC. Constictic, nortictic and stictic acids are the major components in L. pulmonaria; in addition connorstictic, cryptostictic, and menegazziaic acids are present as minor constituents. In $L$. scrobiculata constictic, stictic, and usnic acids and $\mathrm{m}$ - scrobiculin are the major compounds; while cryptostictic and norstictic acids, and p-scrobiculin are present as minors.

\section{Einleitung}

Flechten (Lichenes) sind keine einheitlichen Lebewesen, wie andere Pflanzen. Sie bestehen mindestens aus zwei verschiedenen Lebewesen, die als ein Pilz (Mycobiont) und eine Grün -oder Blatalge (Photobiont) in engem Kontakt zusammenleben (Symbiose). Als primären Photobiont besitzt Lobaria scrobiculata (Scop.) DC eine Blaualge und Lobaria pulmonaria (L.) Hoffm. eine Grünalge.

Die letztere Flechte wurde seit dem Mittelalter als 'Sticta pulmonaria' bekannt. Die aus dieser Flechte hergestellte Droge wurde jahrhundertelang bei einigen Lungenkrankheiten verabreicht. In der Homöopathie wird die aus frischer Flechte bereitete Essenz $\left(D_{1}-\varnothing\right)$ bei Tracheitis, Rhinitis und Rheuma angewendet (1,2). Von Nord-Europa bis zum Mittelmeergebiet kommen folgende fünf Lobaria Arten vor: Lobaria amplissima (Scop.) Forss., L. linita (Ach.) Rabenh., L. pulmonaria (L.) Hoffm., L. scrobiculata (Scop.) DC und L. virens (With.) Laundon. In der Türkei wurden bisher alle diese Arten, mit Ausnahme von $L$. linita nachgewiesen. In den letzten Jahrzehnten hat die Erforschung der Flechtenflora der Türkei grosse Fortschritte gemacht $(3,4)$. NO- Anatolien ist artenreich an Flechten, weil hier ein euxinisches Klima herrscht. Bei einer Exkursion durch dieses Gebiet wurden zwei Lobaria Arten gesammelt und phytochemisch untersucht.

\section{Material und Methode}

Das Untersuchungsmaterial wurde im Juli 1997 in Çamlıhemşin: Zilkale, ca.700 m bei A8- Rize gesammelt.

Die Flechtenstoffe sind sehr oft im Mark (Medula) gelagert .Bei der Laubflechte führt man wie üblich ist, den Schnitt zur Prüfung der Markreaktion von der Lagerunterseite her aus.

Die in dieser Arbeit verwendete Reagenzien sind (7):

1.Kalilauge (Abkürzung : $\mathbf{K}^{\prime}$ )

2.Natriumhypochlorit : (C)

3.Para-Phenylendiamin: (P)

4.Kaliumjodit-Jod Lösung: (J) 
Für anatomische Beobachtungen werden die Lager mindestens einen Tag in der Mischung von Ethylalkohol und Glycerin (1:1) geweicht und anschliessend mit einer Rasierklinge geschnitten.

Die Proben wurden mittels Hochauflösender Flüssigkeitschromatographie (HPLC) auf aromatische Sekundärstoffe untersucht. Dazu wurden $20 \mathrm{mg}$ Flechtenmaterial zermörsert und mit Aceton ca. $1 \mathrm{~h}$ extrahiert. Die Extrakte wurden filitriert für die HPLC-Anaylse verwendet.

Die HPLC wurde nach der Standardmethode der Gradienten-Elution nach Feige et al. (8) durchgeführt. Die benutzte Apparatur (Fa. Kontron) ist mit einer Spherisorb 5 ODS $(2,5 \mu \mathrm{m}, 250 \mathrm{x}$ $4,6 \mathrm{~mm}$ ) Säule ausgestattet. Es werden zwei Laufmittel benutzt. Als Laufmittel A dient Aqua bidest. mit 1\%iger Orthophosphorsäure und als Laufmittel B Methanol (Baker). Die Gradientenelution der Proben erfolgt bei einer Flußrate von $0,7 \mathrm{ml} / \mathrm{min}$. Zu Beginn des Laufes beträgt der Anteil an Laufmittel B 30\%. Nach 1 min isokratischen Laufes werden die Proben injiziert, in den folgenden 14 min steigt der Anteil des Laufmittels B linear von 30 auf $70 \%$ an. Der weitere Anstieg auf 100\% erfolgt in $30 \mathrm{~min}$. Nach einem 18 minutigen isokratischen Lauf endet das Programm. Die eluierten Substanzen werden von einem Detektor bei $\lambda=245 \mathrm{~nm}$ erfaßt und von den auftretenden Peaks jeweils UV-Spektren ( $\lambda=200-400 \mathrm{~nm})$ aufgenommen.

Dem Lösungsmittel Aceton sind $20 \mathrm{mg} / \mathrm{l}$ Solorinsäure als interne Standards beigefügt. Die Inhaltsstoffe werden über eine relativ Retentionszeit und die interne Standard Solorinsäure ermittelt. Diese Relativ Retentionszeit ist stabil, unabhängig vom Alter der Säule.

RRT $=\frac{\text { RT Komponent }}{\text { RT Interne Standard }} \times 100$

Die Auswertung der Ergebnisse wurde mittels des Computerprogramms Wintabolites (9) und der Analyse der UV-Spektren (10) durchgeführt.

\section{Ergebnisse und Diskussion}

\section{Beschreibung der untersuchten Arten}

Genus: Lobaria (Schreber) Hoffm. gehört zu den Laubflechten, die grosse Lager haben.Ihre ca. 60 Arten kommen in warm- und kühl- gemässigten Zonen der Erde vor.

Die Lager sind gelbgrünlich bis olivenbraun. In trockenem Zustand graubräunlich gefärbt. Die Lagerunterseite ist mit kurzen Rhizinen bedeckt. Die Hymenien der Apothecien sind bräunlichrot.

\section{Lobaria scrobiculata (Scop.) DC.}

Sin.: L. verrucosa (Hudson) Hoffm.

Lager mit Blaualgen, in trockenem Zustand blass gelblich, blass gelbgrünlich, grau oder graugelblich; im feuchten Zustand bleigrau. Oberseite flachgrubig, an den Ränden und auf den Rippen sind rundliche Sorale vorhanden. Die Unterseite des Lagers ist mit kurzen Rhizinen bedeckt. Lagerslappen abgerundet und 1-3 cm breit. Apothecien sehr spärlich.

Reaktionen mit Reagenzien : Mark $\mathrm{P}+$ orange, $\mathrm{K}+$ gelb, $\mathrm{C}-$.

Lobaria pulmonaria (L.) Hoffm.

Hauptalge des Lagers eine Grünalge, in trockenem Zustand nicht blass gelblich und im feuchten Zustand nicht grau wie L. scrobiculata, sondern grünlicholiv. Oberseite des Lagers grubignetzribbig. Auf den erhobenen Netzrippen und Ränden des Lagers sind gelbweissliche Sorale oder schuppige Isidien vorhanden.Unterseite des Lagers von schattigen Formen ist etwas glänzend, hell- 
bis schwarzbraun gefärbt und mit kurzen Rhizinen bedeckt.Lagerslappen sind $1-3 \mathrm{~cm}$ breit und an der Spitze gestutzt. Apothecien sehr selten.

Reaktionen mit Reagenzien:

Mark P + rot, $\mathrm{K}+$ gelblichorange, $\mathrm{C}$ -

Tabelle: 1 DieVerteilung der Substanzen von aufgearbeiteten Lobaria pulmonaria. RRT (relative Retentionszeit) wurde laut Interner - Standard Solorinsäure - RT berechnet. Alle Komponenten des Chromatogramms wurden als einzel - Standard Komponent eingespritzt und danach durch Vergleichen der Retentionszeiten identifizient.

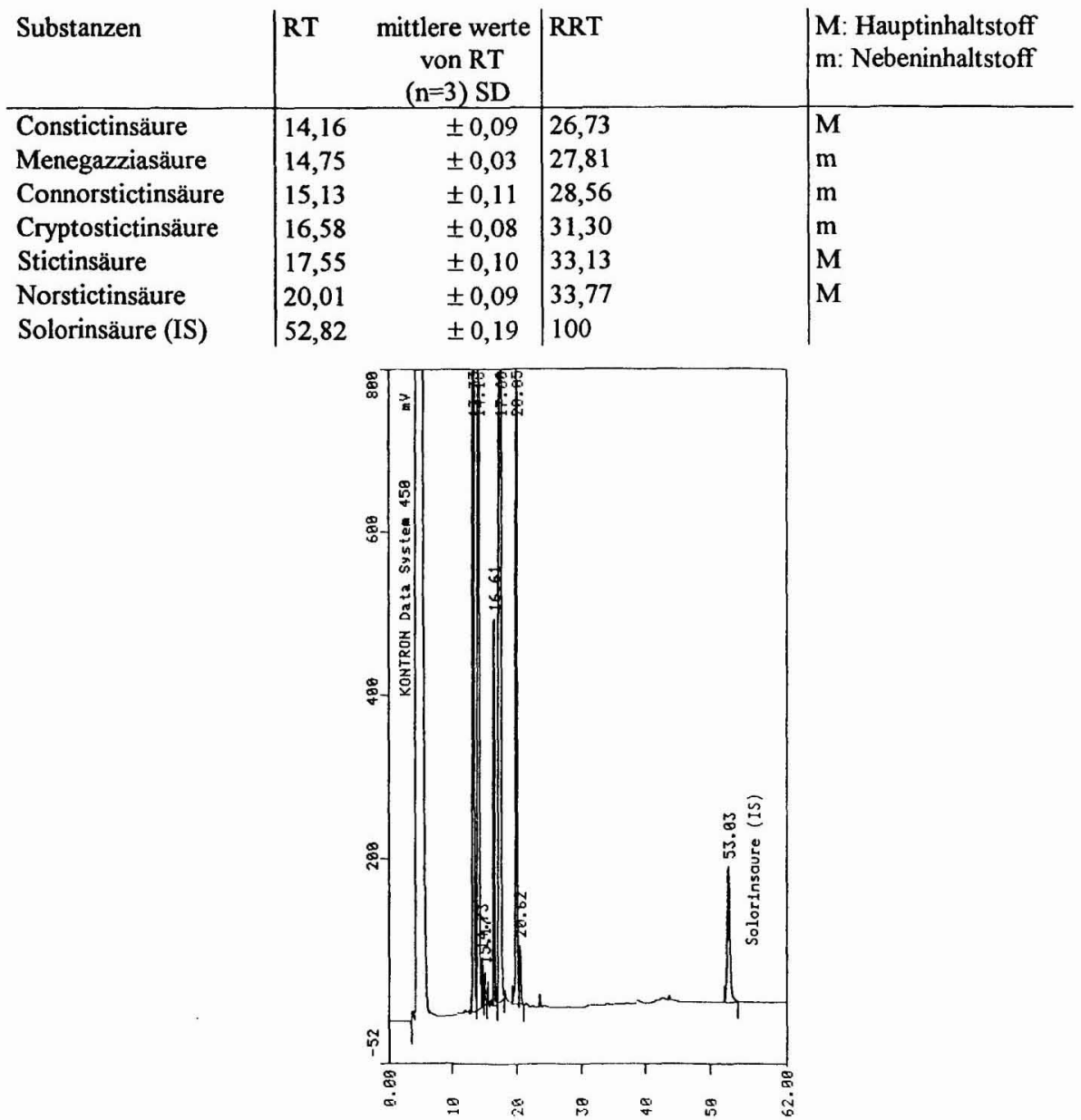

Abbildung 1: HPLC- Trennung der Einzelkomponenten von Lobaria pulmonaria und als interne Standard Solorinsäure (chromatographische Daten siehe Kapitel Material und Methode) 
Tabelle: 2 DieVerteilung der Substanzen von aufgearbeiteten Lobaria scrobiculata. RRT (Relative Retentionszeit) wurde laut Internen - Standard Solorinsäure - RT berechnet. Alle Komponenten des Chromatogramms wurden als einzel - Standard Komponent eingespritzt und danach durch Vergleichen der Retentionszeiten identifizient.

\begin{tabular}{l|lc|l|l} 
Substanzen & RT & $\begin{array}{c}\text { mittlere werte } \\
\text { von RT } \\
(\mathrm{n}=3) \text { SD }\end{array}$ & RRT & $\begin{array}{l}\text { M: Hauptinhaltstoff } \\
\text { m: Nebeninhaltstoff }\end{array}$ \\
\hline Constictinsäure & 14,11 & $\pm 0,05$ & 26,71 & $\mathrm{M}$ \\
Cryptostictinsäure & 16,55 & $\pm 0,04$ & 31,34 & $\mathrm{~m}$ \\
Stictinsäure & 17,44 & $\pm 0,03$ & 33,02 & $\mathrm{M}$ \\
Norstictinsäure & 19,93 & $\pm 0,05$ & 37,73 & $\mathrm{~m}$ \\
m-Scrobiculin & 29,48 & $\pm 0,08$ & 55,81 & $\mathrm{~m}$ \\
p- Scrobiculin & 31,13 & $\pm 0,09$ & 58,88 & $\mathrm{M}$ \\
Usninsäure & 34,20 & $\pm 0,10$ & 64,75 &
\end{tabular}

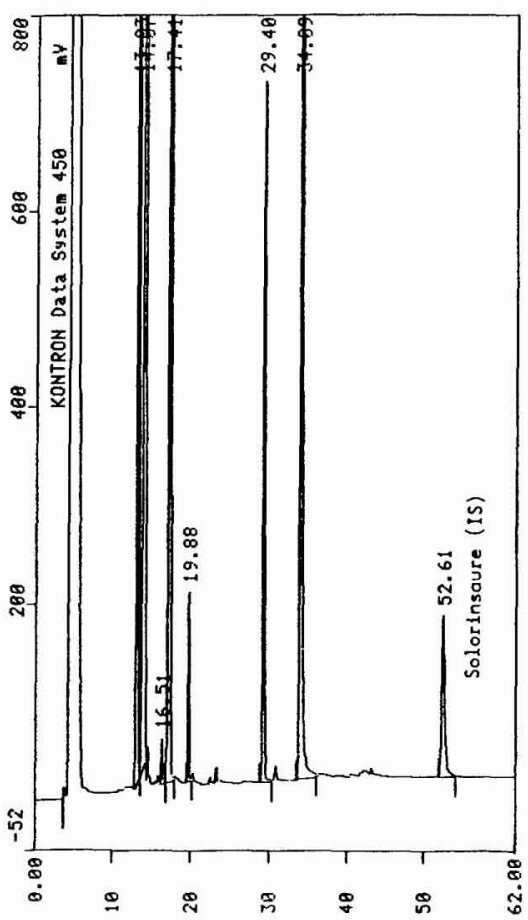

Abbildung 2: HPLC- Trennung der Einzelkomponenten von Lobaria scrobiculata und als interne Standard Solorinsäure (chromatographische Daten siehe Kapitel Material und Methode). 


\section{Diskussion:}

Lobaria scrobiculata und L. pulmonaria unterscheidet man auch nach ihren aromatischen Stoffen (Tab.1,2). Bei den Proben von NO-anatolischen L.pulmonaria wurde keine Usninsäure festgestellt, welche besonders fur die antibiotische Wirkung verantwortlich ist. L. scrobiculata dagegen besitzt als Hauptinhaltsstoff Usninsäure und $\mathrm{m}$-Scrobiculin. Die beide Substanzen fehlen bei L. pulmonaria und p-Scrobiculin wurde nur bei der $L$. scrobiculata festgestellt. Menegazzia- und Connorstictinsäuren sind bei der L. pulmonaria nur in Spuren vorhanden, diese beiden Verbindungen fehlen bei L.scrobiculata.

\section{Danksagung:}

Ich danke den Herren Prof. Dr. G.B. Feige und PD Dr. H. Thorsten Lumbsch (beide Essen) für ihre Hilfe bei der HPLC-Analyse der Proben und Durchsicht des Manuskriptes.

\section{Literatur}

1. Zeybek, U.,. John, V. : Pharmacia, 32 (1), 37-48, (1992)

2. Speta, F.: Flechten Bedrohte Wunder der Natur, S.40, Landesverlag Linz, (1986)

3. John, V.: Das die Türkei Betreffende Lichenologische Schriftum-I, POLLICHIA, Bad Dürkheim, Neunkirchener Druckerei \& Verlag-Neunkirchen (1992)

4. John, V.: Ergänzungen zum die Türkei Betreffenden Likenologischen Schrifttum -IV, POLLICHIA, Bad Dürkheim, Neunkirchener Druckerei \& Verlag Neunkirchen (1995).

5. Wirth, V.: Die Flechtenflora Baden-Württembergs, 2.Aufl. Teil 1-2, Verlag Eugen Ulmer, Stuttgart (1995)

6. Poelt,J., Vezda, A. : Bibl. Lichenol. 16:1 (1981).

7. Zeybek,U., John, V., Lumbsch, H.T.: Doğa-Tr. J. of Botany 17, S. 109-116 (1993)

8. Feige, G.B., Lumbsch, H.T., Huneck, S. \& Elix, J.A.: J. Chromatogr. 646: 417-427. (1993)

9. Mietzsch, E., Lumbsch, H.T. \& Elix, J.A.: Wintabolites (Mactabolites for Windows) . Users, Manual, Essen (1989)

10. Hale, M.E.. Science 123: S.671 (1956)

Anschrift der Verfasser : Ege Universităt, Fakultăt für Pharmazie, Abteilung für Pharmazeutische Botanik, 35100 Bornova-Izmir, Türkei 